\title{
Hawking radiation with angular momentum and the entropy variation in a Kerr black hole
}

\author{
Xin-Yang Wang ${ }^{\mathrm{a}}$, Wen-Biao Liu ${ }^{\mathrm{b}}$ \\ Department of Physics, Beijing Normal University, Beijing 100875, China
}

Received: 24 September 2018 / Accepted: 12 May 2019 / Published online: 17 May 2019

(C) The Author(s) 2019

\begin{abstract}
Considering Hawking radiation with angular momentum, we propose a modified Stefan-Boltzmann law about the particles with both energy and angular momentum radiated from a Kerr black hole. Based on the modified Stefan-Boltzmann law, we calculate the entropy in the interior volume of the Kerr black hole and investigate the proportional relation between the variation of this entropy and the variation of Bekenstein-Hawking entropy under Hawking radiation. Comparing to Hawking radiation without angular momentum, we find that whether the particles radiated from the black hole take angular momentum cannot affect the proportional relation between the two types of entropy. Furthermore, the proportionality coefficient of the two types of entropy is investigated. It is found that this coefficient is approximately a constant except the late stage of Hawking radiation. Moreover, the proportional relation can degenerate to the Schwarzschild case when the angular momentum of the black hole completely disappears. For a Kerr black hole, the extremal black hole can technically not prevent and stop the evaporation anymore under Hawking radiation with angular momentum.
\end{abstract}

\section{Introduction}

It has been shown that the interior volume of a Schwarzschild black hole can increase linearly with the advanced Eddington time [1]. Subsequently, the interior volume definition was extended to the Reissner-Nordstrm and Kerr black holes [2,3], and the results show that the interior volume of these black holes also increases with the advanced time. Besides, to solve the information paradox of black hole, the entropy of quantum field modes in the interior volume of a Schwarzschild black hole has been calculated [4]. It is found that this entropy also increases linearly with the advanced

\footnotetext{
a e-mail: xinyang_wang@foxmail.com

b e-mail: wbliu@bnu.edu.cn
}

time because the entropy is proportional to the interior volume. Moreover, the entropy is found to be proportional to the Bekenstein-Hawking entropy during the black hole evaporation. Subsequently, using this method, the entropy of quantum field modes in the interior volume of a high-dimensional Schwarzschild black hole, a Reissner-Nordstrm black hole and a Kerr black hole has been calculated in Refs. [5-7].

In the previous works [4-7], all the particles radiated from the black hole are uncharged and without angular momentum during Hawking radiation. However, this treatment oversimplifies the process of black hole evaporation, and the extremal black hole will appear and stop the evaporation process in the end technically because of cosmic censorship conjecture. Based on the theory that Hawking radiation is caused by the vacuum fluctuation near the event horizon, Hiscock and Weems [8] have investigated the evaporation process of the charged black hole, and have derived the charge loss rate of the black hole during the evaporation process using the Schwinger formula in quantum field theory. Meanwhile, the Stefan-Boltzmann law is modified to describe both the energy and charge loss rate of a black hole under Hawking radiation, which in the Eddington-Finkelstein coordinates can be expressed as

$$
\frac{d M}{d v}=-\sigma T^{4} A+\frac{Q}{r_{+}} \frac{d Q}{d v} .
$$

However, comparing with charged Hawking radiation, the radiation with angular momentum has not been found yet. Now, analogous to the method in Ref. [8], we will derive a new modified Stefan-Boltzmann law qualitatively, it can be used to investigate the particles with both energy and angular momentum radiated from a Kerr black hole. And then, using the modified Stefan-Boltzmann law, we will investigate the connection between the variation of quantum field entropy inside a Kerr black hole and the variation of BekensteinHawking entropy under Hawking radiation carrying both energy and angular momentum. 
The organization of the paper is as follows. In Sect. 2, we review the interior volume of a Kerr black hole. After that, we qualitatively construct the modified Stefan-Boltzmann law for the particles with both energy and angular momentum radiated from the black hole. Moreover, using the modified Stefan-Boltzmann law, the entropy of the quantum field modes in the interior volume of the Kerr black hole is calculated and the proportional relation is investigated between the variation of this entropy and the variation of Bekenstein-Hawking entropy under Hawking radiation with angular momentum. Considering Hawking radiation without angular momentum, we recalculate the proportional relation and compare the results with the case with angular momentum. The paper ends with discussions and conclusions in Sect. 3 .

\section{Entropy variation under Hawking radiation with angular momentum}

According to Ref. [1], the interior volume of a Kerr black hole in the Eddington-Finkelstein coordinates can be expressed as

$V_{\Sigma}=2 \pi v f_{\max }(M, a)$,

where

$$
\begin{aligned}
f_{\text {max }}(M, a)= & \sqrt{2 M r-r^{2}-a^{2}} \\
& \times\left.\left(\sqrt{r^{2}+a^{2}}+\frac{r^{2}}{2 a} \ln \frac{\sqrt{r^{2}+a^{2}}+a}{\sqrt{r^{2}+a^{2}}-a}\right)\right|_{r=r_{s}} .
\end{aligned}
$$

Here, $v$ is the advanced Eddington time, $a=\frac{J}{M}$ is the angular momentum of unit mass of the black hole, and $f_{\max }(M, a)$ is the maximal value of function $f(r, M, a)$ when the coordinate $r$ takes the special value $r_{s}$.

From Eq. (2), we can see that the interior volume of a Kerr black hole is proportional to the advanced time $v$. It means that, analogous to a Schwarzschild black hole, the interior volume of a Kerr black hole can also increase with the advanced time. This property of the interior volume can influence the statistical properties of quantum field modes in the volume. Naturally, the statistical entropy of the quantum field modes in the interior volume will increase. Besides, the information loss of black hole during Hawking radiation can also be reflected by Bekenstein-Hawking entropy [911]. If we can find the relation between the entropy of the quantum field and the Bekenstein-Hawking entropy, and figure out how it varies with Hawking radiation, the variation of interior (volume) and exterior (horizon) entropy of the black hole can be connected. This is probably a way to solve the information paradox. So, it is significant to calculate the entropy of quantum field modes in the interior volume of the black hole and investigate the connection between this entropy and Bekenstein-Hawking entropy. In the following, we only think about the massless scalar field inside a Kerr black hole.

According to Refs. [4,7], the entropy of the scalar field in the interior volume of a Kerr black hole is

$S_{\Sigma}=\frac{\pi^{2}}{45 \beta^{3}} V_{\Sigma}$

where $\beta$ is the inverse temperature and $V_{\Sigma}$ is the interior volume. It is shown that the entropy increases with the advanced time because it is proportional to the interior volume of the black hole. Equation (4) means that the interior volume can directly influence the statistical properties of the quantum field modes.

In Ref. [7], we have proposed two essential assumptions as the black-body radiation and the quasi-static process. According to the two assumptions, the temperature of the scalar field can be regarded as Hawking temperature. The inverse of temperature $\beta$ can be expressed as

$\beta=\frac{1}{T}=\frac{2 \pi\left[\left(M+\sqrt{M^{2}-a^{2}}\right)^{2}+a^{2}\right]}{\sqrt{M^{2}-a^{2}}}$.

Subsequently, we want to investigate the entropy variation of the scalar field in the interior volume under Hawking radiation. Considering the particles radiated from a Kerr black hole with both energy and angular momentum, the StefanBoltzmann law should be modified. Thinking of the dimensions, it can be directly modified as

$\frac{d M}{d v}=-\sigma T^{4} A+P_{\Omega}$,

where $P_{\Omega}$ is the radiation power of angular momentum. However, how can we obtain the concrete expression for the radiation power of angular momentum with a reasonable physical explanation? Fortunately, combining both the quantum principle of Hawking radiation and the classical idea of Penrose process, the concrete expression of $P_{\Omega}$ can be obtained qualitatively and naturally.

Hawking radiation can be explained as that due to the particle-antiparticle pairs produced by vacuum fluctuation near the event horizon, the antiparticles with negative energy fall into the black hole, and it causes the black hole to lose mass. In the meantime, according to the conservation of total energy, the particles with positive energy will carry the reduced mass of the black hole to escape from the event horizon and travel to infinity. So, for the stationary observer at infinity, this process means that the black hole has just emitted particles with energy and lost mass. 
If considering Hawking radiation with both energy and angular momentum, the particle-antiparticle pairs produced by vacuum fluctuation near the event horizon should be regarded as carrying both energy and angular momentum. However, based on the principle of Hawking radiation, there is an important problem that whether the particle with both energy and an arbitrary angular momentum can propagate from the event horizon to infinity. According to Ref. [12], for Kerr black holes, when a particle with both energy and angular momentum near the event horizon can propagate to infinity, the value of the angular momentum of the particle should be in a specific interval. In other words, for a particle with both energy and an arbitrary angular momentum near the event horizon, it does not necessarily propagate to infinity. Therefore, for Hawking radiation with both energy and angular momentum, we only consider the situation that the particles produced by vacuum fluctuation can propagate from the event horizon to infinity. It is because that a Kerr black hole reduces the mass and angular momentum due to Hawking radiation only if the particles with both mass and angular momentum radiated from the black hole are detected by a stationary observer at infinity.

Therefore, according to the above discussions and Penrose process, the antiparticles carrying both negative energy and angular momentum with the opposite direction as the black hole's, will fall into the black hole along the negative energy orbit in the ergosphere, and it can reduce the mass and angular momentum of the black hole. Due to the conservation of total energy and angular momentum, the particles carrying both positive energy and angular momentum with the same direction as the black hole's, will take along the reduced energy and angular momentum of the black hole away from the ergosphere and travel to infinity. The stationary observer at infinity can regard this total process as that the black hole radiates particles and does work on them, while the energy and angular momentum of the black hole decrease. For the radiation process of energy, it still satisfies the simple Stefan-Boltzmann law, so we just need to find the concrete expression for the radiation power of angular momentum $P_{\Omega}$. According to the above statement, the radiation process of angular momentum can be regarded as that the black hole does work on the outgoing particles, so the radiation power of angular momentum $P_{\Omega}$ can be written as $P_{\Omega}=\Omega \times M$, where $\Omega$ is the angular velocity of the radiated particle, $M$ is the torque of the particle, which can be expressed as the rate of angular momentum changing with the advanced time $\frac{d J}{d v}$. Because the radiation particles are produced by vacuum fluctuation near the event horizon, the angular velocity of the particles can be viewed as the angular velocity of the event horizon. Since the angular velocity of the event horizon is $\frac{a}{r_{+}^{2}+a^{2}}$, the modified Stefan-Boltzmann law can be expressed as $\frac{d M}{d v}=-\sigma T^{4} A+\frac{a}{r_{+}^{2}+a^{2}} \frac{d J}{d v}$

where $\sigma$ is a positive constant related to the number of quantized matter fields coupling with gravity [13], $A$ is the area of the event horizon, and $r_{+}$is the radius of the event horizon. From Eqs. (1) and (7), the correction terms in the modified Stefan-Boltzmann laws are the loss rate of charge and angular momentum of the black hole under Hawking radiation, which are the same as the $d Q$ and $d J$ terms in the first law of thermodynamics of black hole respectively. So the modified Stefan-Boltzmann law can be seemingly regarded as consistent with the first law of thermodynamics of black hole. Substituting the temperature $T$, the area of horizon $A$, and the radius of horizon $r_{+}$into Eq. (7), we have

$$
\begin{aligned}
d v= & -\frac{16 \pi^{3} M^{2}\left(\sqrt{M^{2}-a^{2}}+M\right)^{2}}{\sigma\left(a^{2}-M^{2}\right)^{2}} \\
& \times\left[2 M\left(\sqrt{M^{2}-a^{2}}+M\right) d M-a d J\right] .
\end{aligned}
$$

Next, we want to construct the connection between the entropy of the scalar field in the interior volume of the Kerr black hole and the Bekenstein-Hawking entropy. In Ref. [7], the reasonable relation was given between the two types of entropy using the derivation form. Here, the proportional relation in derivation form between the two entropies can also be obtained in an infinitesimal evaporation process. According to the quasi-static assumption, the variations of both the interior volume and the entropy only depend on the variation of advanced time. Hence, while taking the derivative of the interior volume Eq. (2) and the entropy Eq. (4), the mass and charge can be regarded as constants technically except the late stage of black hole evaporation.

Based on above statement, taking the derivative of two sides of Eq. (2) with respect to advanced time $v$ and substituting Eq. (8) into it, the derivation form of the interior volume can be obtained as

$$
\begin{aligned}
\dot{V}_{\Sigma}= & -\frac{32 \pi^{4} f_{\max }(M, a) M^{2}\left(\sqrt{M^{2}-a^{2}}+M\right)^{2}}{\sigma\left(a^{2}-M^{2}\right)^{2}} \\
& \times\left[2 M\left(\sqrt{M^{2}-a^{2}}+M\right) \dot{M}-a \dot{J}\right],
\end{aligned}
$$

where the dot indicates derivative by $v$. Substituting both Eqs. (5) and (9) into Eq. (4), the derivation form of the scalar field entropy in the interior volume of the Kerr black hole can be written as

$$
\begin{aligned}
\dot{S}_{\Sigma}= & -\frac{\pi^{3} f_{\max }(M, a)}{90 \sigma M \sqrt{M^{2}-a^{2}}\left(\sqrt{M^{2}-a^{2}}+M\right)} \\
& \times\left[2 M\left(\sqrt{M^{2}-a^{2}}+M\right) \dot{M}-a \dot{J}\right],
\end{aligned}
$$

where $\dot{M}<0$ and $\dot{J}<0$. 
Subsequently, the connection between the entropy of the scalar field in the interior volume of the Kerr black hole and the Bekenstein-Hawking entropy can be constructed. The Bekenstein-Hawking entropy is defined as [14-16]

$S_{B H}=\frac{A}{4}$.

Taking the derivative of this equation, we have

$\dot{S}_{B H}=\frac{2 \pi}{\sqrt{M^{2}-a^{2}}}\left[2 M\left(\sqrt{M^{2}-a^{2}}+M\right) \dot{M}-a \dot{J}\right]$.

Combining Eq. (10) with Eq. (11), the proportional relation in derivation form between the entropy inside the Kerr black hole and the Bekenstein-Hawking entropy can be expressed as

$\dot{S}_{\Sigma}=-\frac{\pi^{2}}{180 \sigma} F(M, a) \dot{S}_{B H}$,

where

$F(M, a)=\frac{f_{\max }(M, a)}{M\left(\sqrt{M^{2}-a^{2}}+M\right)}$.

Finally, we consider the particles radiated from Kerr black holes only with energy. It means that the parameter $M$ changes with Hawking radiation and the parameter $J$ should be regarded as a constant. In this case, the mass loss rate of the black hole should satisfy the simple Stefan-Boltzmann law, which can be given as [17]

$\frac{d M}{d v}=-\sigma T^{4} A$.

Repeating the above derivation process, the proportional relation in derivation form between the entropy of the scalar field inside the Kerr black hole and the Bekenstein-Hawking entropy can also be obtained as

$\dot{S}_{\Sigma}=-\frac{\pi^{2}}{180 \sigma} F(M, a) \dot{S}_{B H}$,

where

$F(M, a)=\frac{f_{\max }(M, a)}{M\left(\sqrt{M^{2}-a^{2}}+M\right)}$.

This is an intriguing result that whether the particles radiated from a Kerr black hole with angular momentum cannot influence the proportional relation of the two types of entropy. Actually, for any infinitesimal evaporation process, the proportionality coefficient only depends on the parameters $M$ and $a$ of a Kerr black hole. It should be emphasized that the parameters $M$ and $a$ in function $F(M, a)$ can only be regarded as constants in the infinitesimal process, and their values are the initial values at the beginning of this infinitesimal process. Meanwhile, according to the result, we can find out that the modified Stefan-Boltzmann law should be consistent with the first law of black hole thermodynamics.

\section{Discussions and conclusions}

Although Eq. (16) is same as Eq. (13), they reflect the relation between the entropy in the interior volume of the Kerr black hole and the Bekenstein-Hawking entropy during two different processes of Hawking radiation. Equation (16) shows the proportional relation between the two types of entropy under Hawking radiation carrying only energy, and the independent variable of function $F(M, a)$ is $M$ while $a$ can be considered as a constant. In this situation, when $M$ decreases to $a$, the extremal black hole appears in the evaporation process. The cosmic censorship conjecture suggests that the extremal black hole cannot be achieved. Hence, Hawking radiation will be terminated as the black hole approaches to the extremal black hole. However, Eq. (13) gives us the proportional relation between the two types of entropy under Hawking radiation with both energy and angular momentum, and both $M$ and $a$ are regarded as independent variables of function $F(M, a)$. In this case, the process of black hole evaporation cannot be terminated by the extremal black hole, and it can evaporate more generally.

Based on the detailed calculation, $F(M, a)$ is shown in Fig. 1. At each special value of $a$, the evolution of function $F(M, a)$ can be divided into two stages. In the first stage, the trajectory of $F(M, a)$ remains almost flat until the mass $M$ drops to a "turning interval". It indicates that the entropy of the scalar field in the interior volume of a Kerr black hole is approximately proportional to the Bekenstein-Hawking entropy during the infinitesimal evaporation process in this

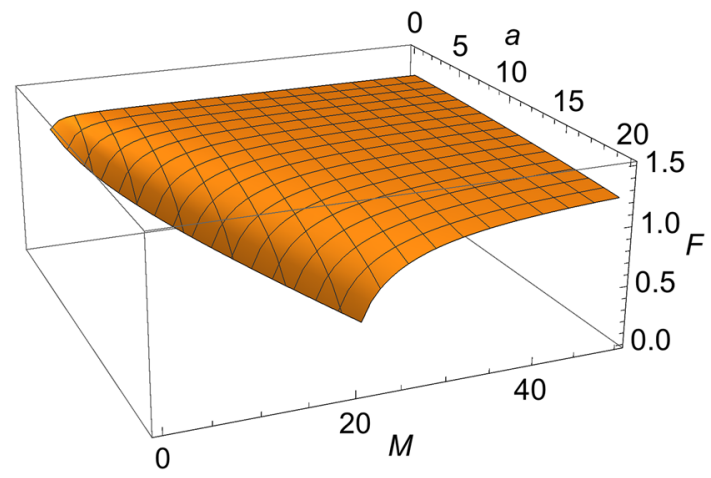

Fig. 1 Plot of $M$ and $a$ versus $F(M, a)$ 
stage. In the second stage, the trajectory of $F(M, a)$ rapidly declines and is terminated. The termination point of the trajectory represents the Kerr black hole becomes an extremal black hole under Hawking radiation. According to cosmic censorship conjecture, this evaporation process is reasonable and the terminating is natural. Besides, the length of flatness of the trajectory increases as the angular momentum of the black hole decreases in the first stage. When the angular momentum of the black hole disappears, the proportional relation of the two types of entropy in the Kerr case can degenerate to the Schwarzschild case, while the entropy of the scalar field in the interior volume of the black hole is always proportional to the Bekenstein-Hawking entropy during Hawking radiation. It illustrates that, in the case of particles with both energy and angular momentum radiated from the black hole, the black hole evolves more freely and generally during the evaporation process. This result is different from the case carrying only energy while the Hawking radiation will be terminated inevitably by the extremal black hole. However, according to Ref. [7], in the degenerating process as Schwarzschild case, the method of derivative form cannot be used, because the evaporation process in this stage cannot be regarded as a quasi-static process. Therefore, at the end of black hole evaporation, using the derivative form to obtain the proportional relation is meaningless.

Besides, the minus sign in Eqs. (12) and (15) illustrates that the entropy variation of the scalar field inside the black hole is opposite to Bekenstein-Hawking entropy variation under Hawking radiation. Since the entropy of the scalar field inside a Kerr black hole is proportional to the interior volume of the black hole, and the interior volume increases with the advanced time, so the scalar field entropy also increases with the advanced time. On the contrary, Bekenstein-Hawking entropy decreases with Hawking radiation. According to the above mentioned result, the variation of the two types of entropy is proportional to each other under Hawking radiation approximately except the late stage of the evaporation process. It can be considered that the variation of the scalar field entropy in the interior volume has a particularly close connection to the variation of Bekenstein-Hawking entropy. Since the information of black hole can be reflected by Bekenstein-Hawking entropy, when Bekenstein-Hawking entropy decreases with Hawking radiation, the information of black hole changes. However, according to the above statement, the entropy of the scalar field inside the black hole increases with the advanced time, and its variation is approximately proportional to the variation of Bekenstein-Hawking entropy under Hawking radiation. A natural viewpoint is that the lost information of the black hole under Hawking radiation may be considered to be stored in the interior volume of the black hole. Therefore, this perspective is a possible way to resolve the information paradox of the black hole.
Till now, we have proposed and applied the modified Stefan-Boltzmann law of a Kerr black hole to describe the Hawking radiation, which reflects the particles radiated with both energy and angular momentum. On this basis, the proportional relation in derivative form between the entropy of the scalar field in the interior volume of the Kerr black hole and the Bekenstein-Hawking entropy has been obtained. Subsequently, when the particles radiated from the black hole only carry energy, the proportional relation in derivative form is naturally given. The results show that whether the particles radiated from the black hole carry angular momentum cannot influence the proportional relation. Finally, the proportional relation of the two types of entropy is analyzed. The entropy of the scalar field in the interior volume of the Kerr black hole is approximately proportional to the BekensteinHawking entropy in an infinitesimal process except the late stage of black hole evaporation. When the angular momentum becomes zero, the proportional relation will degenerate to the Schwarzschild case. It means that the evaporation process of a Kerr black hole is not always terminated by an extremal black hole under charged Hawking radiation. Therefore, in this situation, the black hole can evolve more freely and generally.

Acknowledgements The authors thank Dr. Xiao-Kai He and Dr. ChunYen Lin for so many times of useful discussions. This work is supported by the National Natural Science Foundation of China (Grant no. 11235003).

Data Availability Statement This manuscript has no associated data or the data will not be deposited. [Authors' comment: The paper has no associated data as it is a theoretical paper.]

Open Access This article is distributed under the terms of the Creative Commons Attribution 4.0 International License (http://creativecomm ons.org/licenses/by/4.0/), which permits unrestricted use, distribution, and reproduction in any medium, provided you give appropriate credit to the original author(s) and the source, provide a link to the Creative Commons license, and indicate if changes were made. Funded by SCOAP $^{3}$.

\section{References}

1. M. Christodoulou, C. Rovelli, Phys. Rev. D 91(6), 064046 (2015)

2. Y.C. Ong, Gen. Relativ. Gravit. 47(8), 88 (2015)

3. I. Bengtsson, E. Jakobsson, Mod. Phys. Lett. A 30(21), 1550103 (2015)

4. B. Zhang, Phys. Rev. D 92(8), 081501 (2015)

5. J.Z. Yang, W.B. Liu, Phys. Lett. B 782, 372 (2018)

6. S.Z. Han, J.Z. Yang, X.Y. Wang, W.B. Liu, Int. J. Theor. Phys. 57(11), 3429 (2018)

7. X.Y. Wang, J. Jiang, W.B. Liu. Class. Quantum Gravity 35(21), $215002(2018)$

8. W.A. Hiscock, L.D. Weems, Phys. Rev. D 41, 1142 (1990)

9. D.N. Page, Phys. Rev. Lett. 71, 3743 (1993)

10. D. Marolf, Rep. Prog. Phys. 80(9), 092001 (2017)

11. T. Jacobson, D. Marolf, C. Rovelli, Int. J. Theor. Phys. 44, 1807 (2005) 
12. A.A. Grib, Y.V. Pavlov, JETP Lett. 92, 125 (2010). https://doi.org/ 10.1134/S0021364010150014. arXiv:1004.0913 [gr-qc]

13. L.E. Parker, D. Toms, Quantum Field Theory in Curved Spacetime: Quantized Field and Gravity (Cambridge University Press, Cambridge, 2009)

14. S.W. Hawking, Commun. Math. Phys. 43, 199 (1975) Erratum: [Commun. Math. Phys. 46, 206 (1976)]
15. J.D. Bekenstein, Lett. Nuovo Cim. 4, 737 (1972)

16. J.D. Bekenstein, Phys. Rev. D 7, 2333 (1973)

17. I. Montvay, E. Pietarinen, Phys. Lett. B 110, 148 (1982) 